\title{
CBIR Evaluation using Different Distances and DWT
}

\author{
Omprakash Yadav \\ Assistant Professor, \\ Computer Department, \\ Xavier Institute of Engineering, \\ Mahim, Mumbai, India
}

\author{
Vaishali Suryawanshi \\ Assistant Professor, \\ Computer Department, \\ Thadomal Shahani Engineering \\ College, \\ Bandra, Mumbai, India
}

\begin{abstract}
In this paper, evaluation is made on the result of CBIR system based on haar wavelet transform with different distances for similarity matching to calculate the deviation from query image. So, different distances used are Chessboard distance, Cityblock distance and Euclidean distance. In this paper discrete wavelet transform is used to decompose the image. The image is decomposed till sixth level and last level approximate component is saved as feature vector. Comparison is made between different distances to see the best suited distance for CBIR. The wavelet used is "Haar". Haar has compact support and it is the simplest orthogonal wavelet. It is very fast also.
\end{abstract}

\section{General Terms}

Average Precision, Average Recall, Query Image, Database, Similarity Measurement.

\section{Keywords}

CBIR, QBIC, Precision, Recall, Query Image, Distances, Efficiency, HAAR, Chessboard Distance, City Block Distance, Euclidean Distance.

\section{INTRODUCTION}

An image retrieval system is effective if it can retrieve an image or a collection of images from an operating database upon being inputted with a single image whose replicas or lookalikes need to be extracted. Database management and Computer vision are two major research communities, which study the subject of image retrieval from different perspectives. Text based image retrieval employs techniques of attaching text or data along with the image to describe it, often termed as 'metadata', while content based techniques use visual features to match images to the query image.

In CBIR, all the images from the database are taken and features of each are extracted and stored in a vector. These features are compared to the extracted features of the query image. A CBIR typically converts images in feature vector representations and uses them to match similar images [1].

IBM was the first research company to take initiative by proposing a system called QBIC (query by image content), which was developed, at the IBM Almaden Research Center. Unlike keyword based system, visual features are extracted from images itself. Content based image retrieval system uses contents and extracts features like color, shape and texture. All these are visual contents [2].

Feature extraction based on the color is done taking color histogram of each image. It is nothing but the portion of pixels within an image which has some specific value. This specific value people express as colors. One more benefit of using extraction based on colors is that it does not depend on the size of image. Eventually, color histograms will be taken and compared [3].

Feature extraction based on shape does not refer to the shape of the whole image. Within an image we have certain area of interest. Shape denotes the shape that area of interest. To get the shape first image segmentation is performed or edge detection is done [3].

Feature extraction based on texture measurely concentrates on visual patterns and how they are organized. It is given by texels. Texels are kept into number of different sets ,depending how many of textures detected. The sets determined like this will give complete information about the which patterns and where in the image they got detected. The determination of specific texture can be made by molding texture as a 2D gray level variation. For determination of level of contrast, regularity, directionality and coarseness pixels relative brightness is considered. In this paper texture features are extracted using DWT transform [3].

Figure shows a general description of a standard image retrieval system.

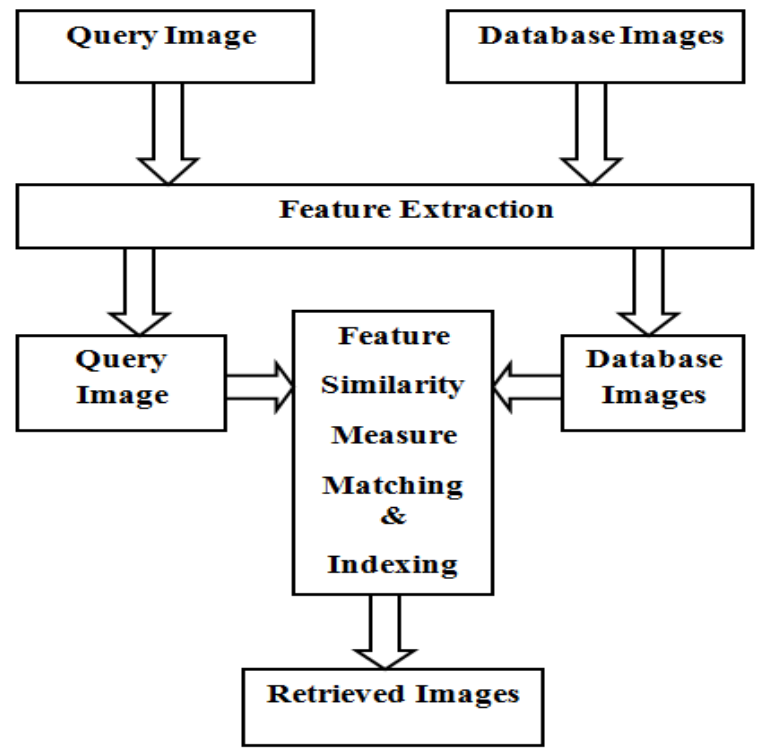

Figure 1. Basic block diagram of CBIR system

As shown in the above diagram, feature extraction is done for both query image as well as database images. First of all, all the databases images will be presented and there feature will be extracted and stored as feature database. Then query image is selected and its features will be extracted. This process will result into query features. After this process, query feature is 
taken and all the database feature is selected, and depending on criteria similarity measure will be calculated and the retrieved images will be displayed. In this paper, similarity measurement criteria is taken based on different distances like Euclidean, chessboard and city block distance [3].

\section{SIMILARITY MEASUREMENT USING DIFFERENT DISTANCES}

The parameters being used for calculation of efficiency of the CBIR system designed, namely Average Precision and Average Recall are the ones being used to compare the results of different Distance Types being employed to retrieve images in the proposed system. Precision and recall can be calculated as follows:

$$
\begin{aligned}
& \text { Precision }=\frac{\text { Numberof releventimagesretrieved }}{\text { Totalnumberof imagesretrieved }} \\
& \text { Recall }=\frac{\text { Numberof releventimagesretrieved }}{\text { Totalnumberof releventimagesinthedatabase }}
\end{aligned}
$$

\subsection{Chessboard Distance}

It is also known as chebyshev distance. It is called chessboard distance, its taken from the chess game. In this game the minimum number of steps taken by a king to move from one square to another square on chessboard equals the Chebyshev distance [3]. Chessboard distance can be calculated as follows: If $\mathrm{A}(\mathrm{x}, \mathrm{y})$ and $\mathrm{B}(\mathrm{p}, \mathrm{q})$ are two pixels then chessboard distance can be given as,

Distance $=\max (|x-p|,|y-q|)$.

\subsection{City Block Distance}

The value of city block distance is always greater than or equal to 0 . We will get 0 if there are identical points under considerations [3]. Value will be high if low similarity is there. City block distance can be calculate as follows:

If $\mathrm{A}(\mathrm{x}, \mathrm{y})$ and $\mathrm{B}(\mathrm{p}, \mathrm{q})$ are two pixels then cityblock distance can be given as, Distance $=|x-p| \oplus|y-q|$.

\subsection{Euclidean Distance}

Euclidean distance metric was initially used by CANDID. He used this for the comparison of global signatures. Euclidean distance can be calculate as follows: If $\mathrm{A}(\mathrm{x}, \mathrm{y})$ and $\mathrm{B}(\mathrm{p}, \mathrm{q})$ are two pixels then euclidean distance can be given as,

$$
\text { Distance }=\sqrt{\left(|x-p|^{2} \oplus|y-q|^{2}\right)} .
$$

\section{DWT LITERATURE REVIEW}

DWT is an mathematical tool ,it decomposes the image into various parts and decomposition is performed hierarchically. It is very much useful in the processing of non-stationary signals. It uses wavelets. Wavelets are small waves and of limited duration. In Fourier transform we loose the time information after transform is performed. But, I $\mathrm{n}$ wavelet transform we get both time and frequency information. Different wavelets can be generated by translation and dilations performed on mother wavelet.
DWT decomposes the image into components like horizontal denoted by $\mathrm{cH}$, vertical component denoted by $\mathrm{cV}$ and diagonal component denoted by $\mathrm{cD}$. Figure below shows the decomposition upto three level. In our project we have used decomposition till sixth level. Major benefit we get by decomposing till sixth level is that feature vector size gets reduced too much [4].

Wavelet transform is very efficient. It can also be implemented using filter banks using convolution. Image processed with wavelet transform gives multi resolution description of image. Image can be viewed at different levels of resolution. In the lowest band LL magnitude is largest level wise. Significantly its good to have more magnitude. Larger the magnitude of wavelet coefficient, it will become more significant [5][6].

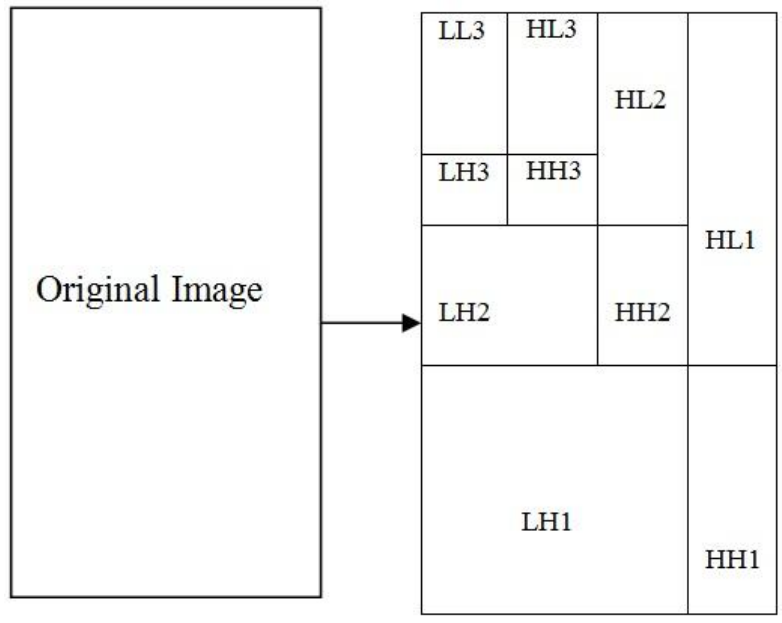

Figure 2: DWT decomposition of image till $3^{\text {rd }}$ level

\section{FIGURES/CAPTIONS}

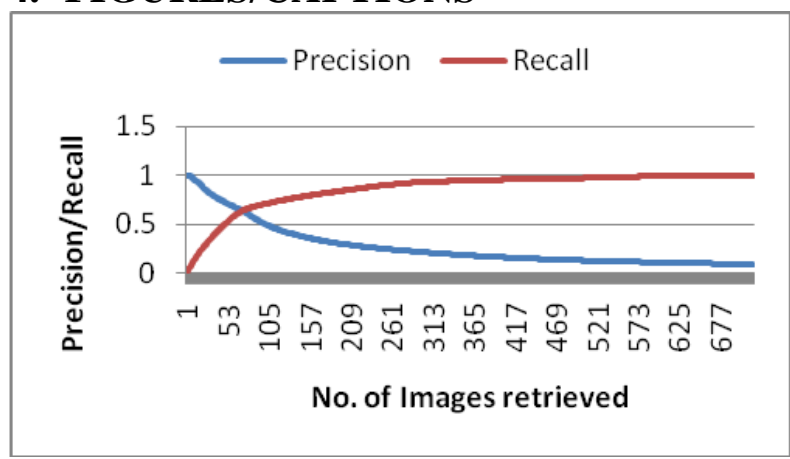

Figure 3. Retrieval using Haar on 720 images(Cityblock Distance)(0.6477) 


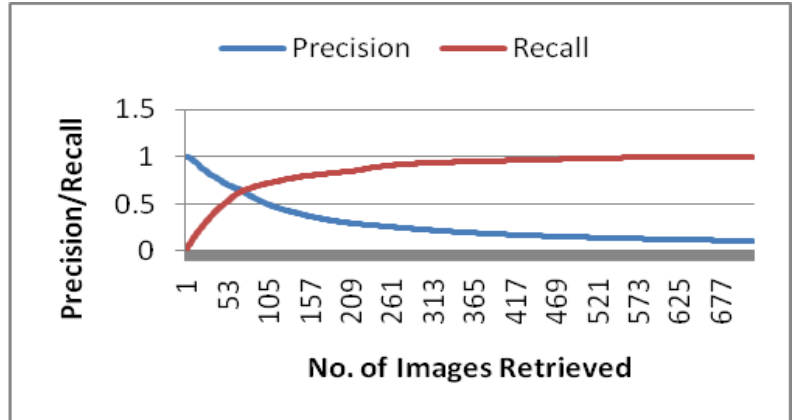

Figure 4. Retrieval using Haar on 720 images(Euclidean Distance)(0.6394)

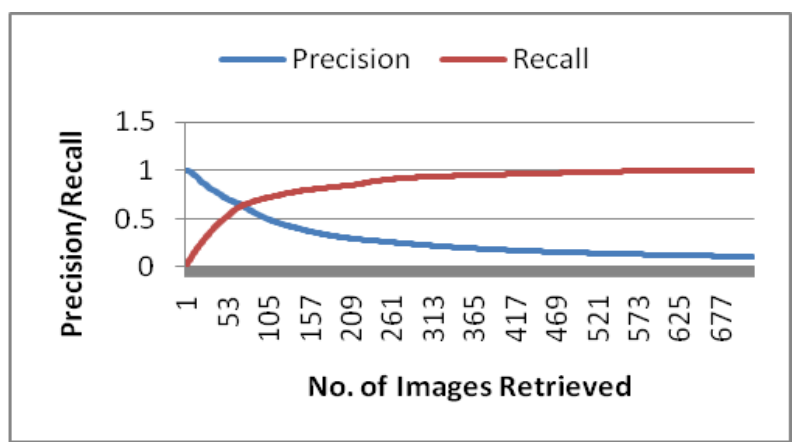

Figure 5. Retrieval using Haar on 720 images(Chessboard Distance)(0.6213)

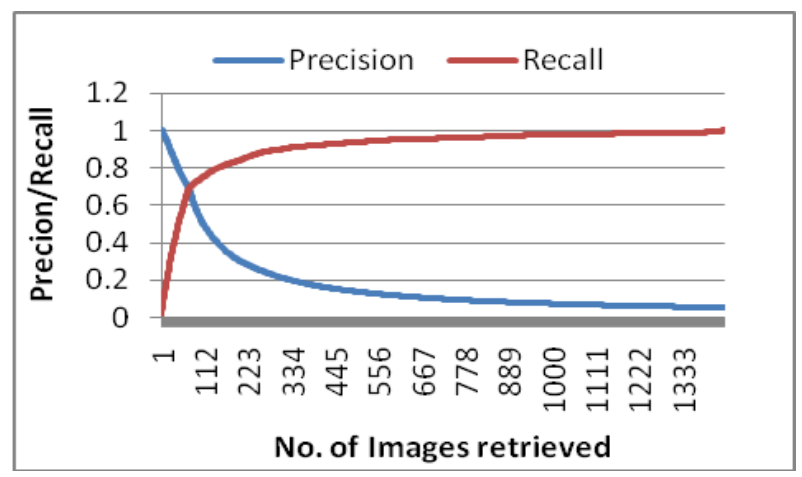

Figure 6. Retrieval using Haar on 1440 images(Euclidean Distance)(0.6884)

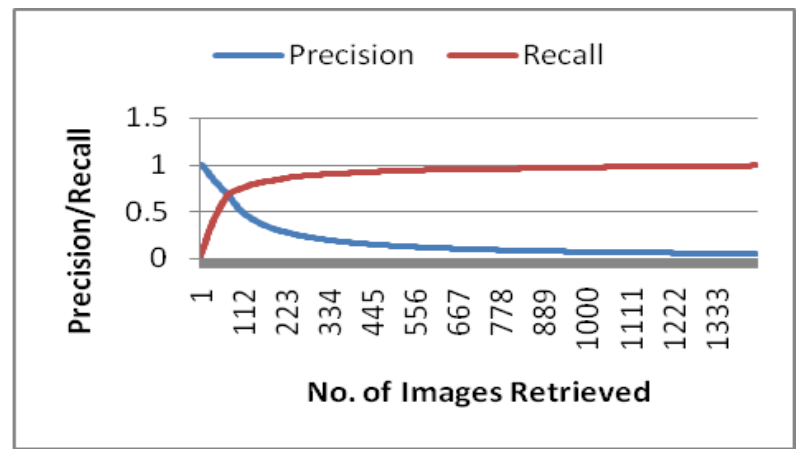

Figure 7. Retrieval using Haar on 1440 images(Cityblock Distance)(0.6872)

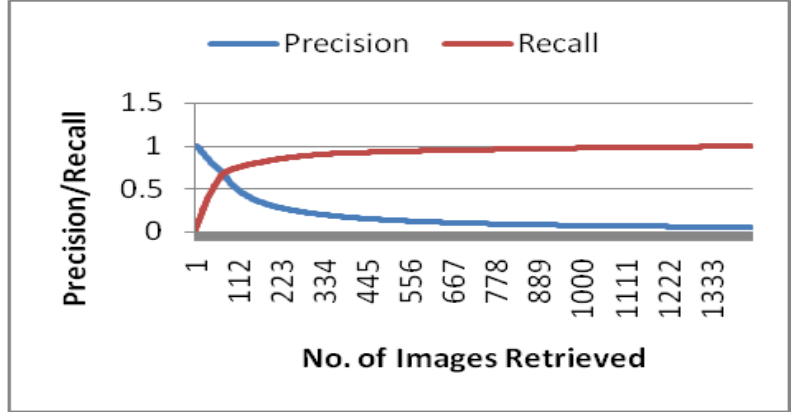

Figure 8. Retrieval using Haar on 1440 images(Chessboard Distance)(0.6816)

\section{SCHEME OF IMPLEMENTATION}

We aim to extract texture from an image. Texture is a pattern on image which can be smooth, rough, etc. The wavelet transformations transform the images into a multi-scale representation with both spatial and frequency characteristics. In this paper feature extraction is done using wavelet transform [5].

\subsection{Feature extraction of database images}

For this project discrete wavelet transform is used to decompose the images. DWT decomposes the image into four parts. Here, decomposition is performed till sixth level.

Step1: Apply first level decomposition and decompose image into 4 subparts i.e. $\mathrm{cA} 1, \mathrm{cH} 1, \mathrm{cV} 1$ and $\mathrm{cD} 1$.

Step2: Apply second level decomposition and again decompose cA1 into four subparts i.e. $\mathrm{cA} 2, \mathrm{cH} 2, \mathrm{cV} 2$ and cD2.

Step3: Apply third level decomposition and again decompose cA2 into its 4 subparts cA3, cH3, cV3 and cD3.

Step4: Apply fourth level decomposition and again decompose cA3 into its 4 subparts $\mathrm{cA} 4, \mathrm{cH} 4, \mathrm{cV} 4$ and $\mathrm{cD} 4$.

Step5: Apply fifth level decomposition and again decompose cA4 into its 4 subparts cA5, cH5, cV5 and cD5.

Step6: Apply sixth level decomposition and again decompose cA5 into its 4 subparts cA6, cH6, cV6 and cD6.

Step7: Save the cA6 as a feature vector.

Where,

$$
\begin{aligned}
& \mathrm{cA}=\text { Approximate coefficient matrix } \\
& \mathrm{cH}=\text { Horizontal component } \\
& \mathrm{cV}=\text { Vertical component } \\
& \mathrm{cD}=\text { Diagonal component }
\end{aligned}
$$

Final feature vector size is $2 * 2 * 3$ after the sixth level decomposition. Do this (step1 to step7) for all the image in the database and store the feature vector of all the images. Here, COIL database is used, of that 720 images is selected of 10 different classes for the first experiment and for second experiment 1440 images of 20 different classes is selected.

\subsection{Feature extraction of query image} After the all images of database is converted into feature vector, query image is taken for retrieval purpose. In this scheme five different query images are taken from each different classes.

Step1: Five query images selected from one type of class 
Step2: Each query image is taken one by one and decomposed using dwt till sixth level and query image feature vector extracted.

Step3: Similarity measurement is done.

Step4: Step 1 to 3 is repeated till all the classes are covered.

Step5: Precision and recall calculated for each query image class wise.

Step 6: Average precision and recall calculated for all the classes [6][7].

\section{RESULTS}

First experiment is carried out on 720 images of 10 different classes of COIL database. In this first experiment cityblock distance gave better results than others.

Using chessboard distance as a similarity matching, it gives only $62.13 \%$ approximately $62 \%$ average precision and recall. This suggests that the Chessboard Distance Type is suitable for efficient implementation of CBIR.

Using euclidean distance as a similarity matching, it gives $63.94 \%$ approximately $64 \%$ average precision and recall.

Using cityblock distance as a similarity matching, it gives $64.77 \%$ approximately $65 \%$ average precision and recall. City Block Distance results indicate a slightly better efficiency, as compared to Chessboard Distance and Euclidean distance. After running CBIR algorithm based on Haar wavelet and using the three major Distance Calculation techniques, the final results clearly indicate that Euclidean Distance and cityblock distance provides good results.

The results of first experiment is tabulated below:

Table 1.Using haar wavelet with combination of different distances on 720 Images

\begin{tabular}{|c|c|c|}
\hline Distance Type & $\begin{array}{c}\text { Average } \\
\text { Precision }\end{array}$ & Average recall \\
\hline $\begin{array}{c}\text { City Block } \\
\text { Distance }\end{array}$ & $64.77 \%$ & $64.77 \%$ \\
\hline $\begin{array}{c}\text { Euclidean } \\
\text { Distance }\end{array}$ & $63.94 \%$ & $63.94 \%$ \\
\hline $\begin{array}{c}\text { Chessboard } \\
\text { Distance }\end{array}$ & $62.13 \%$ & $62.13 \%$ \\
\hline
\end{tabular}

Second experiment is carried out on 1440 images of 20 different classes of COIL database. In this experiment Cityblock distance and Euclidean distance gives approximately same precision and recall. With Chessboard result is marginally lower than Cityblock and Euclidean distance.

The results of second experiment is tabulated below:
Table 2.Using haar wavelet with combination of different distances on 1440 Images

\begin{tabular}{|c|c|c|}
\hline Distance Type & $\begin{array}{c}\text { Average } \\
\text { Precision }\end{array}$ & Average recall \\
\hline $\begin{array}{c}\text { Euclidean } \\
\text { Distance }\end{array}$ & $68.84 \%$ & $68.84 \%$ \\
\hline $\begin{array}{c}\text { City Block } \\
\text { Distance }\end{array}$ & $68.72 \%$ & $68.72 \%$ \\
\hline $\begin{array}{c}\text { Chessboard } \\
\text { Distance }\end{array}$ & $68.16 \%$ & $68.16 \%$ \\
\hline
\end{tabular}

\section{CONCLUSION}

The comparison of distances used for Similarity Measures namely Euclidean Distance, Chessboard Distance and City Block Distance clearly indicates that Euclidean Distance and Cityblock distance provides the good precision and recall value and is thus the best choice for implementing the most efficient version of the proposed CBIR system. For CBIR any of the two Euclidean distance or Cityblock distance can be taken for similarity matching. In this paper experiment is carried out on the same database i.e. COIL. In future scope, this experiment can be carried out on different and more natural databases for example corel database and with these three different distances.

\section{REFERENCES}

[1] Lidia Xavier, Thusnavis Bella Mary.I and Newton David Raj.W, "Content Based Image Retrieval Using Textural Features based on Pyramid structure wavelet transform", 2011 IEEE.

[2] Priyanka P. Buch, Madhuri V. Vaghasia and Sahista M. Machchhar, "Comparative analysis of content based image retrieval using both color and texture", International conference on Current Trends in Technology,2, NUiCONE, 2011 IEEE.

[3] Prabhjot Kaur and Dr. Satish Devane , "Effective method of Cbir for Time and Accuracy", International Journal of Advanced Research in Computer Science and Software Engineering, Volume 3 Issue 11 November 2013.

[4] Vaishali S, Dr. Sachin R. Gengaje , "Literature Review of Wavelet Based Digital Image Watermarking Techniques", International Journal of Computer Applications (0975 - 8887) Volume 31- No.1, October 2011.

[5] Swapnalini Pattanaik and prof.D.G. Bhalke, "Beginners to Content Based Image Retrieval", International Journal of Scientific Research Engineering \& Technology, Volume 1 Issue2 pp 040-044 May 2012.

[6] Arnold W.M. Smeulders, Marcel Worring, Simone Santini, "Content-based image retrieval at the end of the early years".IEEE Transactions on Pattern analysis and machine intelligence, vol 22, No 12, December 2000. 
[7] N S T Sai, R C Patil, "Image Retrieval using 2D DualTree Discrete Wavelet Transform",. International Journal of Computer Applications (0975 - 8887), Volume 14 No.6,February2011.

[8] Elif Albuz, Erturk Kocalar, and Ashfaq A. Khokhar, "Scalable Color Image indexing and Retrieval Using Vector Wavelets", IEEE Transaction on Knowledge and Data engineering, vol. 13, NO. 5, Sep/Oct 2001.
[9] M. Flickner, H. Sawhney, W. Niblack, J. Ashley, Q.Huang, B. Dom, M. Gorkani, J. Hafner, D. Lee, D.Petkovic, D. Steele, and P. Yanker, "Query by Image and Video Content: The QBIC System", IEEE Computer,28(9):23-32,Sept.1995.

[10] N.G. Kingsbury and T. H. Reeves, "Redundantrepresentation with complex wavelets: how to achieve sparsity", in Proc. Int. Conf. Image Processing,Barcelona, Sept. 2003. 